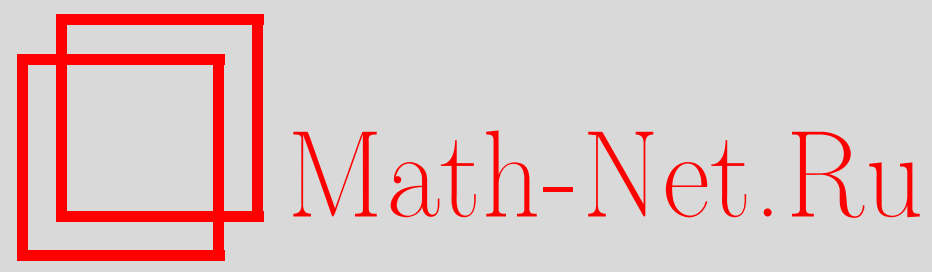

В. В. Картак, О геометрии точечно-инвариантного класса обыкновенных дифференциальных уравнений третьего порядка, Матем. заметки, 2005, том 77, выпуск 5, 719-726

DOI: https://doi.org/10.4213/mzm2527

Использование Общероссийского математического портала Math-Net.Ru подразумевает, что вы прочитали и согласны с пользовательским соглашением http://www.mathnet.ru/rus/agreement

Параметры загрузки:

IP : 54.197 .217 .227

26 апреля 2023 г., 13:08:28

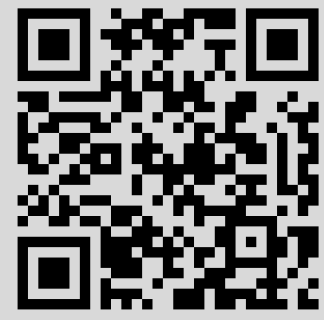




\section{О ГЕОМЕТРИИ ТОЧЕЧНО-ИНВАРИАНТНОГО КЛАССА ОБЫКНОВЕННЫХ ДИФФЕРЕНЦИАЛЬНЫХ УРАВНЕНИЙ ТРЕТЬЕГО ПОРЯДКА}

B. B. Картак

Применены геометрические методы для исследования одного класса обыкновенных дифференциальных уравнений третьего порядка, замкнутого относительно точечных преобразований. Из коэффициентов уравнения сформированы псевдовекторные поля, ассоциированные с уравнением. Записаны условия в виде равенств нулю пяти псевдовекторных полей, при вьполнении которых уравнение обладает максимальной алгеброй точечных симметрий.

Библиограффия: 11 названий.

1. Введение. Идея исследования некоторых свойств дифференциальных уравнений, исследуя связанные с ними геометрические объекты, лежит в основе целого ряда работ, в частности, классических работ по групповому анализу (см. [1]-[3]).

Геометрический подход был применен в работах [4]-[6], где были рассмотрены обыкновенные дифференциальные уравнения второго порядка следующего вида:

$$
y^{\prime \prime}=P(x, y)+3 Q(x, y) y^{\prime}+3 R(x, y) y^{\prime 2}+S(x, y) y^{\prime 3} .
$$

Из коэффициентов $P, Q, R$ и $S$ этих уравнений были образованы геометрические объекты, преобразующиеся при точечных преобразованиях

$$
\left\{\begin{array}{l}
\widetilde{x}=\widetilde{x}(x, y) \\
\widetilde{y}=\widetilde{y}(x, y)
\end{array}\right.
$$

как псевдовекторные и псевдоскалярные поля. На их основе была построена классификация уравнений (1.1) относительно точечных преобразований (1.2).

Аналогичный подход был использован в работе [7] для изучения уравнений точечного расширения класса (1.1)

$$
y^{\prime \prime}=\frac{P(x, y)+4 Q(x, y) y^{\prime}+6 R(x, y)\left(y^{\prime}\right)^{2}+4 S(x, y)\left(y^{\prime}\right)^{3}+L(x, y)\left(y^{\prime}\right)^{4}}{Y(x, y)-X(x, y) y^{\prime}} .
$$

Работа выполнена при финансовой поддержке Российского фонда фундаментальных исследований, гранты № 00-01-00068а и № 02-01-06321мас, и фонда INTAS, грант № 03-51-5007. 
В работе [8] были изучены обыкновенные дифференциальные уравнения третьего порядка, разрешенные относительно старшей производной

$$
y^{\prime \prime \prime}=F\left(x, y, y^{\prime}, y^{\prime \prime}\right)
$$

Относительно специальных точечных преобразований

$$
\left\{\begin{array}{l}
\widetilde{x}=\varphi(x), \\
\widetilde{y}=\psi(x, y)
\end{array}\right.
$$

для уравнений (1.3), обладающих большими групшами точечных симметрий $(4,5,6$ и 7$)$, решена проблема эквивалентности.

В работе [9] проделана классификация линейных обыкновенных дифференциальных уравнений третьего порядка

$$
y^{\prime \prime \prime}=v(x) y^{\prime}+w(x) y
$$

относительно контактных преобразований

$$
\left\{\begin{array}{l}
\widetilde{x}=\widetilde{x}\left(x, y, y^{\prime}\right) \\
\widetilde{y}=\widetilde{y}\left(x, y, y^{\prime}\right) \\
\widetilde{y}^{\prime}=\left(\widetilde{y}_{x}+y^{\prime} \widetilde{y}_{y}\right) /\left(\widetilde{x}_{x}+y^{\prime} \widetilde{x}_{y}\right)
\end{array}\right.
$$

В данной работе мы будем рассматривать следующий класс обыкновенных дифференциальных уравнений третьего порядка:

$$
\begin{aligned}
y^{\prime \prime \prime}= & \frac{-3 X(x, y) y^{\prime \prime 2}+P(x, y) y^{\prime \prime} y^{\prime 2}+Q(x, y) y^{\prime \prime} y^{\prime}+R(x, y) y^{\prime \prime}+S(x, y) y^{\prime 5}}{Y(x, y)-X(x, y) y^{\prime}} \\
& +\frac{L(x, y) y^{\prime 4}+K(x, y) y^{\prime 3}+M(x, y) y^{\prime 2}+N(x, y) y^{\prime}+T(x, y)}{Y(x, y)-X(x, y) y^{\prime}} .
\end{aligned}
$$

В работе [10] показано, что этот класс уравнений является инвариантным классом относительно невырожденньх точечных преобразований (1.2), т.е. любое преобразование (1.2) сохраняет вид уравнения (1.4). Это означает, что применение геометрического подхода для его исследования обосновано.

2. Редуцированное уравнение (1.4). Пусть запись $\Phi_{i, j}$ обозначает частную производную функции $\Phi(x, y)(i+j)$-го порядка $i$ раз по переменной $x$ и $j$ раз по переменной $y: \partial^{i+j} \Phi / \partial x^{i} \partial y^{j}$. Будем трактовать преобразования (1.2) как замену координат $(x, y)$ на плоскости на новые координаты $(\widetilde{x}, \widetilde{y})$. Тогда прямую и обратную матрицы перехода замены координат (1.2) можно записать следующим образом:

$$
\mathbb{S}=\left\|\begin{array}{ll}
x_{1.0} & x_{0.1} \\
y_{1.0} & y_{0.1}
\end{array}\right\|, \quad \mathbb{T}=\left\|\begin{array}{ll}
\widetilde{x}_{1.0} & \widetilde{x}_{0.1} \\
\widetilde{y}_{1.0} & \widetilde{y}_{0.1}
\end{array}\right\| .
$$

Коэффициенты $P, Q, R, S, T, L, K, M, N, X, Y$ уравнения (1.4) меняются под действием преобразований (1.2) согласно некоторым правилам. Будем считать, что для функций $X$ и $Y$, стоящих в знаменателе формулы (1.4), это правило совпадает с правилом 
преобразования вектора с компонентами $(X, Y)$. Этого всегда можно добиться умножением числителя и знаменателя формулы (1.4) на некоторую калибровочную функцию $f(x, y)$, как это было сделано в работе [7].

Выберем систему координат $(\widehat{x}, \widehat{y})$, в которой векторное поле $(X, Y)$ единично:

$$
\widehat{X}=0, \quad \widehat{Y}=1
$$

В новой системе координат уравнение (1.4) упростится, и мы получим редуцированное уравнение (для удобства вычислений, здесь и ниже, вместо переменных $(\widehat{x}, \widehat{y})$ будем писать просто $(x, y))$ :

$$
\begin{aligned}
y^{\prime \prime \prime}= & P(x, y) y^{\prime \prime} y^{\prime 2}+Q(x, y) y^{\prime \prime} y^{\prime}+R(x, y) y^{\prime \prime}+S(x, y) y^{\prime 5} \\
& +L(x, y) y^{\prime 4}+K(x, y) y^{\prime 3}+M(x, y) y^{\prime 2}+N(x, y) y^{\prime}+T(x, y) .
\end{aligned}
$$

Уравнения (2.3) представляют собой инвариантньй класс уравнений относительно точечных преобразований специального вида:

$$
\left\{\begin{array}{l}
\widetilde{x}=h(x), \\
\widetilde{y}=y+g(x) .
\end{array}\right.
$$

Для преобразования координат (2.4) прямая и обратная матрищы перехода (2.1) будут следующими:

$$
\mathbb{S}=\frac{1}{h^{\prime}}\left\|\begin{array}{cc}
1 & 0 \\
-g^{\prime} & h^{\prime}
\end{array}\right\|, \quad \mathbb{T}=\left\|\begin{array}{cc}
h^{\prime} & 0 \\
g^{\prime} & 1
\end{array}\right\| .
$$

Класс (2.3) замечателен также тем, что он содержит все уравнения Пенлеве-типа третьего порядка. Такие уравнения известны как уравнения Шази. Согласно работе [11] все они обязаны иметь следующий вид:

$$
\begin{aligned}
y^{\prime \prime \prime}= & (Q y+A(x)) y^{\prime \prime}+R\left(y^{\prime}\right)^{2}+\left(S y^{2}+B(x) y+C(x)\right) y^{\prime} \\
& +T y^{4}+D(x) y^{3}+E(x) y^{2}+F(x) y+G(x) .
\end{aligned}
$$

3. Преобразования коэффициентов уравнения (2.3) и псевдовекторные поля. Запишем законы изменения коэффициентов уравнения (2.3) относительно преобразований (2.4).

Первая пара коэффициентов $P$ и $Q$, стоящих при старших степенях производных $y^{\prime \prime}$ и $y^{\prime}$, преобразуется по сходным формулам:

$$
P=\frac{1}{h^{\prime}} \widetilde{P}, \quad S=\frac{1}{h^{2}} \widetilde{S}
$$

Следующая пара коэффициентов $Q$ и $L$ также имеет похожие формулы преобразования:

$$
Q=\widetilde{Q}+\frac{2 g^{\prime}}{h^{\prime}} \widetilde{P}, \quad L=\frac{1}{h^{\prime}} \widetilde{L}+\frac{5 g^{\prime}}{h^{\prime 2}} \widetilde{S} .
$$


ОПРЕДЕЛЕНИЕ 1. Поле $\psi=\left(\psi^{1}, \psi^{2}\right)$ есть псевдовекторное поле веса $n$, если при преобразованиях (1.2) с прямой и обратной матрицами перехода $\mathbb{S}$ и $\mathbb{T}(2.1)$ оно трансформируется согласно правилу

$$
\left\|\begin{array}{c}
\psi^{1} \\
\psi^{2}
\end{array}\right\|=(\operatorname{det} T)^{n}\left\|\begin{array}{cc}
S_{1}^{1} & S_{2}^{1} \\
S_{1}^{2} & S_{2}^{2}
\end{array}\right\|\left\|\begin{array}{c}
\widetilde{\psi}^{1} \\
\widetilde{\psi}^{2}
\end{array}\right\| .
$$

В случае преобразований (2.4) правило упростится:

$$
\left\|\begin{array}{c}
\psi^{1} \\
\psi^{2}
\end{array}\right\|=\left(h^{\prime}\right)^{n-1}\left\|\begin{array}{cc}
1 & 0 \\
-g^{\prime} & h^{\prime}
\end{array}\right\|\left\|\begin{array}{c}
\widetilde{\psi}^{1} \\
\widetilde{\psi}^{2}
\end{array}\right\| .
$$

Таким образом, мы можем видеть, что $P$ и $S$ есть псевдоскалярные поля весов -1 и -2 соответственно, а из пар коэффициентов $P$ и $Q, S$ и $L$ можно составить два псевдовекторных поля

$$
\alpha=\left\|\begin{array}{c}
-2 P \\
Q
\end{array}\right\|, \quad \beta=\left\|\begin{array}{c}
-5 S \\
L
\end{array}\right\|
$$

весов 0 и -1 соответственно.

Запишем законы преобразования остальных коэффициентов уравнения (1.4):

$$
\begin{aligned}
R= & h^{\prime} \widetilde{R}+g^{\prime} \widetilde{Q}+\frac{g^{\prime 2}}{h^{\prime}} \widetilde{P}+3 \frac{h^{\prime \prime}}{h^{\prime}} \\
K= & \widetilde{K}+\frac{4 g^{\prime}}{h^{\prime}} \widetilde{L}+\frac{10 g^{\prime 2}}{h^{\prime 2}} \widetilde{S}-\frac{h^{\prime \prime}}{h^{\prime 2}} \widetilde{P} \\
M= & h^{\prime} \widetilde{M}+3 g^{\prime} \widetilde{K}+\frac{6 g^{\prime 2}}{h^{\prime}} \widetilde{L}+\frac{10 g^{\prime 3}}{h^{\prime 2}} \widetilde{S}-\frac{h^{\prime \prime}}{h^{\prime}} \widetilde{Q}+\frac{g^{\prime \prime} h^{\prime}-3 g^{\prime} h^{\prime \prime}}{h^{\prime 2}} \widetilde{P} \\
N= & h^{\prime 2} \widetilde{N}+2 g^{\prime} h^{\prime} \widetilde{M}+3 g^{\prime 2} \widetilde{K}+\frac{4 g^{\prime 3}}{h^{\prime}} \widetilde{L}+\frac{5 g^{\prime 4}}{h^{\prime 2}} \widetilde{S}-\frac{h^{\prime \prime}}{h^{\prime}} \widetilde{R} \\
& +\frac{g^{\prime \prime} h^{\prime}-2 g^{\prime} h^{\prime \prime}}{h^{\prime}} \widetilde{Q}+g^{\prime} \frac{2 g^{\prime \prime} h^{\prime}-3 g^{\prime} h^{\prime \prime}}{h^{\prime 2}} \widetilde{P}+\frac{h^{\prime \prime \prime} h^{\prime}-3 h^{\prime \prime 2}}{h^{2}} \\
T= & h^{\prime 3} \widetilde{T}+g^{\prime} h^{\prime 2} \widetilde{N}+g^{\prime 2} h^{\prime} \widetilde{M}+g^{\prime 3} \widetilde{K}+\frac{g^{\prime 4}}{h^{\prime}} \widetilde{L}+\frac{g^{\prime 5}}{h^{\prime 2}} \widetilde{S} \\
& +\left(g^{\prime \prime} h^{\prime}-g^{\prime} h^{\prime \prime}\right) \widetilde{R}+\frac{g^{\prime}}{h^{\prime}}\left(g^{\prime \prime} h^{\prime}-g^{\prime 2} h^{\prime \prime}\right) \widetilde{Q} \\
& +g^{\prime 2} \frac{g^{\prime \prime} h^{\prime}-g^{\prime} h^{\prime \prime}}{h^{\prime 2}} \widetilde{P}+\frac{g^{\prime} h^{\prime \prime \prime} h^{\prime}+3 g^{\prime \prime} h^{\prime \prime} h^{\prime}-g^{\prime \prime \prime} h^{\prime 2}-3 g^{\prime} h^{\prime \prime 2}}{h^{\prime 2}} .
\end{aligned}
$$

Псевдовекторные поля $\alpha$ и $\beta$ либо тождественно равны 0 в любьх координатах, либо в 0 не обращаются ни в каких координатах. Рассмотрим вырожсденый случай, когда

$$
\alpha=0, \quad \beta=0 .
$$

При условии (3.9) образуем еще одно псевдовекторное поле веса 1

$$
\gamma=\left\|\begin{array}{c}
-3 K \\
M
\end{array}\right\|
$$


Для поля (3.10) также существуют две возможности: либо оно тривиально, либо нет. Допустим, что

$$
\gamma=0
$$

В этом случае редуцированное уравнение (2.3) упрощается и имеет вид

$$
y^{\prime \prime \prime}=R(x, y) y^{\prime \prime}+N(x, y) y^{\prime}+T(x, y) .
$$

Все последующие вычисления относятся к вырожденному случаю, когда выполнены условия (3.9) и (3.11), а уравнение (2.3) свелось к уравнению (3.12).

4. Анализ определяющих уравнений для (3.12). Инфинитезимальньй оператор имеет вид

$$
X=U(x, y) \frac{\partial}{\partial x}+V(x, y) \frac{\partial}{\partial y} .
$$

Он имеет третье продолжение

$$
X_{3}=U(x, y) \frac{\partial}{\partial x}+V(x, y) \frac{\partial}{\partial y}+U_{1}(x, y) \frac{\partial}{\partial y^{\prime}}+U_{2}(x, y) \frac{\partial}{\partial y^{\prime \prime}}+U_{3}(x, y) \frac{\partial}{\partial y^{\prime \prime \prime}}
$$

где

$$
\begin{aligned}
U_{1}(x, y)= & D V(x, y)-y^{\prime} D U(x, y)=V_{1.0}+y^{\prime} V_{0.1}-y^{\prime}\left(U_{1.0}+y^{\prime} U_{0.1}\right) \\
U_{2}(x, y)= & D U_{1}(x, y)-y^{\prime \prime} D U(x, y) \\
= & V_{2.0}+2 y^{\prime} V_{1.1}-y^{\prime} U_{2.0}-2 y^{\prime 2} U_{1.1}+y^{\prime 2} V_{0.2} \\
& -y^{\prime 3} U_{0.2}+y^{\prime \prime} V_{0.1}-2 y^{\prime \prime} U_{1.0}-3 y^{\prime \prime} y^{\prime} U_{0.1}, \\
U_{3}(x, y)= & D U_{2}(x, y)-y^{\prime \prime \prime} D U(x, y) \\
= & 3 y^{\prime} V_{2.1}-y^{\prime} U_{3.0}-3 y^{\prime 2} U_{2.1}+3 y^{\prime 2} V_{1.2}-3 y^{\prime 3} U_{1.2}+3 y^{\prime \prime} V_{1.1} \\
& -3 y^{\prime \prime} U_{2.0}-9 y^{\prime \prime} y^{\prime} U_{1.1}+y^{\prime \prime \prime} V_{0.1}-3 y^{\prime \prime \prime} U_{1.0}-3 y^{\prime \prime 2} U_{0.1}+y^{\prime 3} V_{0.3} \\
& -y^{\prime 4} U_{0.3}-4 y^{\prime \prime \prime} y^{\prime} U_{0.1}-6 y^{\prime 2} y^{\prime \prime} U_{0.2}+3 y^{\prime} y^{\prime \prime} V_{0.2}+V_{3.0} .
\end{aligned}
$$

Уравнение (3.12) определяет многообразие $M$ в пространстве переменных $\left(x, y, y^{\prime}\right.$, $\left.y^{\prime \prime}, y^{\prime \prime \prime}\right)$. Это многообразие инвариантно относительно действия продолженного оператора $X_{3}$ :

$$
\left.X_{3}(M)\right|_{M}=0 \text {. }
$$

После расщепления полученного уравнения по степеням $y, y^{\prime}, y^{\prime \prime}$ получим следующие 9 уравнений:

$$
\begin{aligned}
U_{0.3} & =0 \\
V_{0.3} & =3 U_{1.2}-R U_{0.2} \\
3 V_{1.2} & =3 N U_{0.1}-2 R U_{1.1}+3 U_{2.1}+R V_{0.2} \\
U_{0.2} & =0 \\
3 V_{0.2} & =R U_{0.1}+9 U_{1.1} \\
3 V_{2.1} & =N_{1.0} U+2 N U_{1.0}+4 T U_{0.1}-R U_{2.0}+U_{3.0}-N_{0.1} V+2 R V_{1.1} \\
U_{0.1} & =0 \\
3 U_{2.0} & =-R_{1.0} U-R U_{1.0}-R_{0.1} V+3 V_{1.1} \\
V_{3.0} & =T_{1.0} U+3 T U_{1.0}+T_{0.1} V+N V_{1.0}-T V_{0.1}+R V_{2.0} .
\end{aligned}
$$


Проанализируем систему уравнений (4.1)-(4.9). Уравнения (4.1)-(4.4) есть дифференциальные следствия уравнений (4.5) и (4.7). Таким образом, независимыми являются уравнения (4.5)-(4.9). Причем уравнение (4.5) примет вид

$$
V_{0.2}=0 .
$$

Продифференцируем уравнение (4.8) по переменной $x$ :

$$
3 U_{3.0}=-U R_{2.0}-2 U_{1.0} R_{1.0}-R U_{2.0}-V R_{1.1}-V_{1.0} R_{1.0}+3 V_{2.1} .
$$

Теперь подставим полученное выражениев равенство (4.6). Послеприведения подобных членов новое уравнение примет вид

$$
\begin{aligned}
V_{2.1}= & \left(\frac{1}{2} N_{1.0}+\frac{2}{9} R R_{1.0}-\frac{1}{6} R_{2.0}\right) U+\left(\frac{2}{9} R^{2}-\frac{1}{3} R_{1.0}+N\right) U_{1.0} \\
& +\left(\frac{1}{2} N_{0.1}+\frac{2}{9} R R_{0.1}-\frac{1}{6} R_{1.1}\right) V-\frac{1}{6} R_{0.1} V_{1.0}+\frac{1}{3} R V_{1.1}
\end{aligned}
$$

Правые части уравнений (4.7)-(4.11) зависят только от переменных $U, U_{1.0}, V, V_{1.0}$, $V_{0.1}, V_{2.0}, V_{1.1}$.

5. Условия максимальности алгебры точечных симметрий уравнения (2.3). Составим 7-мерньй вектор $\Psi=\left(U, U_{1.0}, V, V_{1.0}, V_{0.1}, V_{2.0}, V_{1.1}\right)$. Система уравнений (4.7)-(4.11) может быть записана как система уравнений Пфаффа:

$$
\partial_{x} \Psi=\Lambda_{x} \Psi, \quad \partial_{y} \Psi=\Lambda_{y} \Psi .
$$

Условие разрешимости системы уравнений Пфаффа записывается в виде условия коммутирования двух дифференциальных операторов

$$
\left[\partial_{x}-\Lambda_{x}, \partial_{y}-\Lambda_{y}\right]=0 .
$$

Расписывая покомпонентно последнее равенство, получим следующие соотношения между коэффициентами $R, N, T$ уравнения (3.12):

$$
\begin{gathered}
R_{0.1}=0, \quad 3 N+\frac{2}{3} R^{2}-R_{1.0}=0, \\
N_{0.1}=0, \quad T_{0.1}=0 .
\end{gathered}
$$

Под действием преобразований (2.4) равенства (5.1) преобразуются таким образом:

$$
\begin{aligned}
R_{0.1} & =h^{\prime} \widetilde{R}_{0.1} \\
3 N+\frac{2}{3} R^{2}-R_{1.0} & =h^{\prime 2}\left(3 \widetilde{N}+\frac{2}{3} \widetilde{R}^{2}-\widetilde{R}_{1.0}\right)-g^{\prime} h^{\prime} \widetilde{R}_{0.1} .
\end{aligned}
$$

Следовательно, величины из (5.1) являются компонентами псевдовекторного поля $\xi$ веса 2:

$$
\xi=\left\|\begin{array}{c}
R_{0.1} \\
3 N+\frac{2}{3} R^{2}-R_{1.0}
\end{array}\right\| .
$$

Величины из равенства (5.2) преобразуются так:

$$
\begin{aligned}
& N_{0.1}=h^{\prime 2} \widetilde{N}_{0.1}-h^{\prime \prime} \widetilde{R}_{0.1} \\
& T_{0.1}=h^{\prime 3} \widetilde{T}_{0.1}+g^{\prime} h^{2} \widetilde{N}_{0.1}+\left(g^{\prime \prime} h^{\prime 4}-\frac{g^{\prime} h^{\prime \prime}}{h^{\prime}}\right) \widetilde{R}_{0.1} .
\end{aligned}
$$

При вьполнении условия $\xi=0$ из них можно составить псевдовекторноеполе $\eta$ веса 3 :

$$
\eta=\left\|\begin{array}{c}
-N_{0.1} \\
T_{0.1}
\end{array}\right\|
$$


ТЕОремА 1. Уравнение (2.3) имеет 7-мерную алгебру точечных симметрий тогда и только тогда, когда все пять псевдовекторных полей $\alpha, \beta, \gamma, \xi$ и $\eta$ равны 0. Такие уравнения эквивалентны $y^{\prime \prime \prime}=0$ относительно преобразований (2.4).

ДокАЗАТЕЛЬСТВО. Пусть вьполнены условия $\alpha=0, \beta=0, \gamma=0$; тогда уравнение (2.3) примет вид (3.12). Обратное условие также верно, поэтому рассмотрим подробно только уравнения (3.12).

Введем преобразование, обратное преобразованию (2.4):

$$
\left\{\begin{array}{l}
x=p(\widetilde{x}) \\
y=\widetilde{y}+q(\widetilde{y}) .
\end{array}\right.
$$

Если оно переводит уравнение (3.12) в уравнение $y^{\prime \prime \prime}=0$, то обязаны выполняться следующие условия:

$$
\widetilde{R}=0, \quad \widetilde{N}=0, \quad \widetilde{T}=0 .
$$

Используем формулы для преобразования уравнения $y^{\prime \prime \prime}=0$ из работы [10]:

$$
R=-\frac{3 p^{\prime \prime}}{p^{\prime 2}}, \quad N=-\frac{3 p^{\prime \prime}}{p^{\prime 3}}, \quad T=\frac{q^{\prime \prime \prime}}{p^{\prime 3}}
$$

Пересчитаем их в первоначальных переменных

$$
R=\frac{3 h^{\prime \prime}}{h^{\prime}}, \quad N=\frac{h^{\prime \prime \prime} h^{\prime}-3 h^{\prime 2}}{h^{2}} .
$$

По закону дифференцирования сложной функции справедлива следующая формула:

$$
R_{1.0}=3 \frac{h^{\prime \prime \prime} h^{\prime}-h^{\prime \prime 2}}{h^{2}}=3 N+\frac{2}{3} R^{2} .
$$

Следовательно, если наше уравнение (3.12) эквивалентно $y^{\prime \prime \prime}=0$, то тождественно зануляются оба векторных поля $\xi$ и $\eta$.

$\mathrm{C}$ другой стороны, пусть $\xi=0$ и $\eta=0$. Мы определим функцию $h(x)$ как решение дифференциального уравнения $3 h^{\prime \prime}-R h^{\prime}=0$ с некоторыми начальными условиями. Тогда в силу второго соотношения (5.1) формула для коэффициента $N$ из (5.6) верна. Поскольку мы знаем функцию $h(x)$, мы также знаем обратную функцию $p(\widetilde{x})$. Следовательно, мы можем пересчитать коэффициент $T(x)=T(p(\widetilde{x}))$. Тогда из равенства $(5.5)$ мы найдем функцию $q(\widetilde{x})$ и так докажем существование подходящей функции $g(x)$.

Теорема доказана.

Запишем базис полученной 7-мерной алгебры точечных симметрий. Мы можем это сделать разньми способами:

1) $\Psi=(1,0,0,0,0,0,0), X=\frac{\partial}{\partial x}, x \rightarrow x+t, y \rightarrow y$;

2) $\Psi=(0,1,0,0,0,0,0), X=x \frac{\partial}{\partial x}+\frac{\partial}{\partial y}, x \rightarrow x e^{t}, y \rightarrow y$;

3) $\Psi=(0,0,1,0,0,0,0), X=\frac{\partial}{\partial y}, x \rightarrow x, y \rightarrow y+t$; 

4) $\Psi=(0,0,0,1,0,0,0), X=x \frac{\partial}{\partial y}, x \rightarrow x, y \rightarrow y+t x$
5) $\Psi=(0,0,0,0,1,0,0), X=y \frac{\partial}{\partial y}, x \rightarrow x, y \rightarrow y e^{t}$;
6) $\Psi=(0,0,0,0,0,1,0), X=x^{2} \frac{\partial}{\partial y}, x \rightarrow x, y \rightarrow y+t x^{2}$;
7) $\Psi=(0,0,0,0,0,0,1), X=\frac{x^{2}}{2} \frac{\partial}{\partial x}+x y \frac{\partial}{\partial y}, x \rightarrow \frac{2 x}{2+x t}, y \rightarrow \frac{4 y}{2+x t}$.

6. Благодарности. Автор благодарит сотрудников технического университета г. Дрезден (Германия), на базе которого выполнена работа. Персональная благодарность проф. Г. Шайтхауеру за отличные условия, предоставленные для работы.

\section{СПИСОК ЦИТИРОВАННОЙ ЛИТЕРАТУРЫ}

[1] Ibragimov N. Kh. Elementary Lie Group Analisis and Ordinary Differential Equations. Wiley Series in Mathematical Methods in Practice. V. 4. England: John Wiley \& Sons, 1999.

[2] Олвер П. Приложения групп Ли к дифференциальным уравнениям. М.: Мир, 1989.

[3] Бочаров А. В., Вербовецкий А. М., Виноградов А. М. и др. Симметрии и законы сохранения уравнений математической физики. М.: Факториал, 1997.

[4] Dmitrieva V. V., Sharipov R. A. On the point transformations for the second order differential equations // E-print solv-int/9703003, 1997.

[5] Sharipov R. A. On the point transformations for the equation $y^{\prime \prime}=P+3 Q y^{\prime}+3 R y^{\prime 2}+S y^{\prime 3}$ // E-print solv-int/9706003, 1997.

[6] Sharipov R. A. Effective procedure of point classification for the equations $y^{\prime \prime}=P+3 Q y^{\prime}+$ $3 R y^{\prime 2}+S y^{\prime 3} / /$ E-print math.DG/9802027, 1998 .

[7] Михайлов О.Н., Шарипов Р.А. О геометрии точечных расширений одного класса дифференциальных уравнений второго порядка // Дифференц. уравнения. 2000. Т. 36. № 10 . C. $1331-1335$;.

[8] Grebot G. The characterization of third order ordinary differential equations admitting a transitive fiber-preserving point symmetry group // J. Math. Anal. Appl. 1997. V. 206. P. 364-388.

[9] Yumaguzhin V. A. Classification of 3rd order linear ODE up to equivalence // Differential Geom. Appl. 1996. V. 6. P. 343-350.

[10] Дмитриева В.В.Точечно-инвариантные классы ОДУ третьего порядка // Матем. заметки. 2001. Т. 70. № 2. С. 195-200.

[11] Cosgrove C. M. Chazy classes IX-XII of third order differential equations // Research Report \#98-23 (24 June), 1998.

Башкирский государственный университет, г. Уфа 